\title{
Heart failure is not a determinant of central apnea index in the pediatric population
}

\author{
Jonathan Wheeler ${ }^{1}$, Kaylee Tutrow ${ }^{1}$, Eric Ebenroth ${ }^{2}$, Benjamin Gaston ${ }^{3}$, and Anuja \\ Bandyopadhyay $^{2}$ \\ ${ }^{1}$ Indiana University School of Medicine \\ ${ }^{2}$ Riley Hospital for Children at Indiana University Health \\ ${ }^{3}$ Riley
}

September 11, 2020

\begin{abstract}
Background/Objectives: Adults with heart failure (HF) have high prevalence of central sleep apnea (CSA). While this has been repeatedly investigated in adults, there is a deficiency of similar research in the pediatric population. The goal of this study was to compare prevalence of CSA in children with and without HF and correlate central apnea events with heart function. Methods: Retrospective analysis of data from children with and without HF was conducted. Eligible children were $<18$ years old with echocardiogram and polysomnogram within 6 months of each other. Children were separated into groups with and without HF and groups with and without elevated central apnea index (CAI) for comparative study. Results: 120 children (+HF:19, -HF:101) were included. The +HF group was younger, with higher prevalence of trisomy 21, muscular dystrophy, oromotor incoordination, and structural heart disease and lower Apnea Hypopnea Index and lower CAI. Prevalence of CSA was similar in both the groups. LogCAI was inversely correlated to age at time of sleep study. Children with elevated CAI were younger and had higher prevalence of prematurity. There was no difference in left ventricular ejection fraction (LVEF) between groups with and without elevated CAI. Conclusion: In contrast to adults, after adjusting for age, there is no difference in frequency of central apneic events in children with and without heart failure. Unlike in adults, LVEF does not correlate with CAI in children. Overall, it appears that CAI may be more a function of age rather than of heart function in the pediatric population.
\end{abstract}

\section{INTRODUCTION}

In adults with heart failure (HF), central sleep apnea (CSA) is highly prevalent and associated with higher mortality rates ${ }^{1,2}$. HF is defined as the insufficiency of the heart to pump adequate blood to the organs of the body. The etiology of HF in children often differs from adults ${ }^{3}$. In adults, coronary heart disease, hypertension, acquired valvular defects, arrhythmias and myocardial infarction are the leading causes of HF. In children, however, congenital heart defects and the sequelae of time and surgical intervention, and less commonly, cardiomyopathy are the main causes of $\mathrm{HF}^{4}$. While the relationship between HF and CSA has been well studied in adults, there is a lack of similar data in children ${ }^{5}$. Sleep apnea is obstructive (due to airway obstruction) or central in nature (lack of drive to breathe) ${ }^{6}$. The increased risk of CSA in HF is theorized to be due to hemodynamic and autonomic mechanisms involving the sympathetic nervous system's upregulated response to periods of hypoxemia and hypercapnia ${ }^{7}$. While both obstructive sleep apnea (OSA) and CSA can present with increased apneic events, the physiology of OSA and CSA are different, hence treatments differ. In OSA, occlusion of airflow increases cardiac afterload and decreases stroke volume ${ }^{8}$. Relative hypotension triggers baroreceptors to increase sympathetic tone ${ }^{9}$. CSA occurs when partial pressure of $\mathrm{CO}_{2}$ falls below apneic threshold, a level of $\mathrm{CO}_{2}$ below which the nervous system fails to trigger breathing ${ }^{7}$. Patients with HF have a higher ventilatory response due to altered chemosensitivity to increased levels of 
$\mathrm{CO}_{2}{ }^{10}$. Furthermore, decreased cardiac output creates a delay in detection of changes in $\mathrm{CO}_{2}$ levels which exacerbates apnea/hyperventilation cycles ${ }^{11}$. By treating sleep apnea in children and thereby reducing sympathetic output, these adverse effects can be mitigated. However, the link between CSA and HF in children has not been described. This is important to know, as CSA is a potentially reversible condition with which appropriate treatment may improve morbidity and mortality in children with HF.

CSA in infants with congenital heart disease is associated with a fourfold increase in mortality ${ }^{12}$. However, the study describing these findings did not report prevalence of HF or its association with CSA. In children with HF due to dilated cardiomyopathy, CSA was noted in $19 \%$ of the studied population ${ }^{5}$. No correlation was noted between CSA and heart function. In another prospective uncontrolled case series, there was a high prevalence of CSA with significant correlation between left ventricular end diastolic index, left ventricular end systolic index, and central apnea index ${ }^{13}$. However, this study focused on comparing polysomnogram (PSG) indices with heart function based on the nature of cardiomyopathy but did not compare prevalence of CSA in children with and without HF. While there is a known high prevalence of OSA and CSA in adults with HF, this has not been well studied in children. Moreover, no study has been done to correlate central apnea events with heart function, in children with HF. We hypothesized that the prevalence of CSA will be different in children with HF compared to children without HF.

\section{METHODS}

\section{Study Design}

We performed a retrospective, cross-sectional analysis on children with and without HF and compared their demographics, medical comorbidities, PSGs and echocardiograms. Institutional Review Board approval was obtained. Data completed between January 1, 2015 and December 31, 2019 from a single tertiary care center was collected and reviewed.

\section{Participants}

All children (0 - 18 years of age) who had echocardiogram and PSG performed within 6 months of one another were included in the study. Echocardiograms were reviewed by JW and EE and the cohort was divided into groups with $(+\mathrm{HF})$ and without heart failure $(-\mathrm{HF})$. The $+\mathrm{HF}$ group was defined by left ventricular ejection fraction (LVEF) of [?] $45 \%$ or moderate to severe cardiac dysfunction. The -HF group was defined by LVEF of [?]58\% or normal cardiac function and therefore was deemed appropriate for the control group $^{15}$. Values in the intermediate range were excluded to ensure that the population being studied had significant enough cardiac dysfunction to confidently separate them from the controls. Exclusion criteria included structural congenital heart disease with right to left shunting, neurological conditions associated with CSA such as Arnold Chiari malformation, congenital central hypoventilation syndrome, patients on noninvasive ventilation or tracheostomy at the time of PSG, and patients with inadequate sleep time defined by total sleep time less than 120 minutes.

All patient charts with test data in the study time period were reviewed with 50,144 children identified and screened for eligibility. Figure 1 shows the screening process for the study. The initial cohort was screened for individuals with both PSG and echocardiogram within 6 months of each other before being divided into $+\mathrm{HF}$ and -HF groups based on echocardiogram results. To limit the control cohort to a reasonable size, we only included data on children with -HF from January 1, 2019 through December 31, 2019. Exclusion criteria were then applied with 120 patients ultimately confirmed to be eligible and included in the study.

\section{Variables}

Demographic data was collected and included: date of birth, age at time of PSG, age at time of echocardiogram, gender, race, BMI z-score (age $>2$ years) / weight for height z-score (age $<2$ years) at time of echocardiogram, and blood pressure and oxygen saturations closest to echocardiogram date. Data on medical comorbidities was collected based on diagnostic billing codes (ICD-10). Comorbidities studied were prematurity, bronchopulmonary dysplasia, laryngomalacia, craniofacial anomaly, trisomy 21, neuromuscular disorder or Arnold Chiari malformation, hypertension, pulmonary hypertension, feeding dysfunction, and 
obesity. Surgical history was collected on adenoidectomy, tonsillectomy or adenotonsillectomy as well as cardiac surgery and type. Heart defects in all children were noted and broadly classified into two groups: structural heart disease or no structural heart disease.

\section{Polysomnography}

Children underwent PSG at an accredited sleep laboratory as part of clinically indicated care. PSG tests were performed in technical accordance with standards proposed by the American Academy of Sleep Medicine (AASM), by registered polysomnography technicians. Electroencephalogram (placement of frontal [F3,F4], central [C3,C4], occipital [O1,O2] electrodes referenced to the opposite mastoid electrodes [M1,M2]), electrooculogram, electromyogram (chin and both legs), electrocardiogram, pressure transducer and thermistor airflow, uncalibrated respiratory inductance plethysmography, oximetry, and end-tidal $\mathrm{CO}_{2}\left(\mathrm{ETCO}_{2}\right)$ data with video monitoring of the study for scoring support was collected. All studies were scored by a pediatric pulmonologist board certified in sleep medicine, in accordance with pediatric scoring rules proposed by the $\mathrm{AASM}^{16}$. In our institute, post-sigh central apneas are not scored.

Standard PSG collects a number of variables as a multi-parametric test to evaluate and diagnose sleep disorders. PSG data collected included: date of study, age at time of study, percentage of sleep spent in REM, sleep efficiency, Oxygen Desaturation Index (ODI), Arousal Index (AI), Apnea Hypopnea Index (AHI), Central Apnea Index (CAI), Mixed Apnea Index (MAI), Obstructive Apnea Index (OAI), Hypopnea Index (HI), Central Apnea Hypopnea Index (CAHI), average pulse rate, Total Periodic Limb Movement Index, average oxygen saturations in wake vs. sleep, nadir saturations in sleep, total duration of desaturations, total sleep time spent between 45 and $49 \mathrm{mmHg}^{\mathrm{ETCO}_{2}}$, total sleep time spent greater than $50 \mathrm{mmHg} \mathrm{ETCO}_{2}$, average $\mathrm{ETCO}_{2}$ during sleep, and peak $\mathrm{ETCO}_{2}$ during sleep.

\section{Definitions $^{14}$}

ODI - Number of [?] 3\% arterial oxygen desaturations per hour.

AI - Number of arousals/awakenings per hour of sleep.

AHI - Number of apnea and hypopnea events per hour during sleep with apneas defined as a drop in peak excursion of air flow by [?] $90 \%$ of baseline for at least 2 breaths with or without associated desaturation or arousal and hypopneas defined as a drop in peak excursion of air flow [?]30\% for at least 2 breaths with [?] $3 \%$ desaturation from baseline or with associated arousal

CAI - Number of central apnea events per hour during sleep; respiratory event meeting apnea criteria, associated with absent inspiratory effort throughout the entire duration of the event, and at least one of the following is met:

- The event lasts 20 seconds or longer.

- The event lasts at least the duration of two breaths during baseline breathing and is associated with an arousal or [?]3\% oxygen desaturation.

- For infants younger than 1 year of age, the event lasts at least the duration of two breaths during baseline breathing and is associated with a decrease in heart rate to less than 50 beats per minute for at least 5 seconds or less than 60 beats per minute for 15 seconds.

MAI - Number of mixed apnea events per hour during sleep; events meeting apnea criteria for at least the duration of 2 breaths during baseline breathing and is associated with absent respiratory effort during one portion of the event and the presence of inspiratory effort in another portion, regardless of which portion comes first.

OAI - Number of obstructive apnea events per hour during sleep; events meeting apnea criteria for at least the duration of 2 breaths during baseline breathing and is associated with presence of respiratory effort throughout the entire period of absent airflow.

HI - Number of hypopnea events per hour during sleep. 
Pediatric OSA - In children, it is defined as obstructive apnea hypopnea index (OAHI) [?]1 obstructive event per hour with the event being obstructive or mixed apnea or obstructive hypopnea in nature ${ }^{17}$.

AASM CSA - The AASM defines CSA as central apnea hypopnea index (CAI+HI) [?]5/hr. and that the number of central apnea/hypopnea events is $>50 \%$ of total apnea/hypopnea events ${ }^{17}$. This definition is commonly used in the adult population.

Pediatric CSA - Definition of CSA is varied throughout pediatric literature ${ }^{15}$. In order to help facilitate comparison of our results with other pediatric studies on CSA, we defined pediatric CSA as CAI $+\mathrm{HI}>1 / \mathrm{hr}$. and number of central apnea/hypopnea events $>50 \%$ of total apnea/hypopnea events. For the purposes of this study, all CSA referenced will use the Pediatric CSA definition unless otherwise stated.

Elevated CAI - Using the AASM definition of clinically significant pediatric sleep apnea as AHI >1/hr., for the purpose of this study, we defined elevated central apnea index as CAI $>1 / \mathrm{hr}$.

\section{Echocardiogram}

All echocardiograms were performed using a lab standard protocol, on various ACUSON ultrasound machines (Siemens Medical Solutions, Malvern, PA), using probes appropriate for patient size. Images were acquired from parasternal long and short axis views, apical four-chamber and two-chamber views, subcostal, and suprasternal views. Images were transferred to dedicated workstations (Syngo Dynamics, Siemens Medical Solutions, Malvern, PA). All measurements and analyses were performed offline.

Echocardiogram data collected included: date of study, age at time of study, weight $(\mathrm{kg})$, height $(\mathrm{cm})$, body surface area (BSA), right ventricular (RV) size at end diastole $(\mathrm{mm})$, thickness of interventricular septum (IVS) at end diastole $(\mathrm{mm})$, left ventricular $(\mathrm{LV})$ size at end diastole $(\mathrm{mm})$, thickness of left ventricular posterior wall (LVPW) at end diastole ( $\mathrm{mm})$, thickness of IVS at end systole $(\mathrm{mm}), \mathrm{LV}$ size at end systole $(\mathrm{mm})$, thickness of LVPW at end systole $(\mathrm{mm})$, LVEF (Teicholz Formula), visual estimate of RV systolic function, presence and severity of mitral valve stenosis, presence and severity of mitral valve regurgitation, and presence and severity of tricuspid valve regurgitation. To accommodate for difference in ages and thus sizes between the $+\mathrm{HF}$ group and the -HF group and subsequently between the normal and elevated CAI groups, we used the Boston Children's Hospital z-score system for echocardiographic parameters ${ }^{16,17}$. The regressions used included 2D LV End-diastolic Septal Thickness vs BSA, 2D LV End-diastolic Dimension vs BSA, 2D LV End-diastolic Free Wall thickness vs BSA, 2D LV End-systolic Septal Thickness vs BSA, 2D LV End-systolic Dimension vs BSA, and 2D LV End-systolic Free Wall Thickness vs BSA.

\section{Statistical Analysis}

Demographics, clinical parameters, and prevalence of comorbidities between the two groups were analyzed using Mann Whitney U test for continuous variables and Chi-Square tests, with Fisher's Exact test being used to verify results when cell counts were small, for categorical variables. Comparison of polysomnographic parameters was performed between children with and without HF using analysis of covariance (ANCOVA) adjusting for age at time of sleep study. We performed Pearson correlation between OAI as well as CAI with echocardiogram parameters. Furthermore, we analyzed the relationship between LogCAI and age at time of sleep study using Pearson correlation coefficient. Finally, we compared demographic and echocardiographic parameters in children with normal CAI and children with elevated CAI. All analytic assumptions were verified, and all analyses were performed using SPSS v26 statistical software (Armonk, NY: IBM Corp). In this pilot study we sought to determine the relationship between CSA and HF, so sample size was limited to the small number of patients meeting inclusion criteria.

\section{RESULTS}

Table 1 compares age and medical diagnoses between the two groups. The + HF group had a significantly higher prevalence of trisomy 21, muscular dystrophy, and oromotor incoordination. The + HF group also had, as expected, a higher prevalence of structural heart disease than the -HF group. The types of structural heart disease in the + HF group included atrial septal defect (ASD), ventricular septal defect (VSD), coarctation 
of the aorta $(\mathrm{CoA})$, pulmonary stenosis (PS), cardiac fibroma, double outlet right ventricle (DORV), and hypertrophic cardiomyopathy. Children with -HF included ASD, VSD, PS, CoA, DORV, vascular ring, bicuspid aortic valve, and truncus arteriosus.

Table 2 depicts the relationship between PSG and echocardiogram findings between the two groups. The + HF group had lower median AHI and lower median CAI when compared to the -HF group. Furthermore, the + HF group had a higher median 2D LV End-diastolic Septal Thickness vs BSA z-score and a higher median 2D LV End-systolic Dimension vs BSA z-score.

At this point we adjusted for age to see if the observed elevations in central apnea measures were a function of age, rather than heart failure condition. An ANCOVA model was used with dependent variable of AHI, fixed factor of $+\mathrm{HF}$ group, and covariate of age with Bonferroni correction due to the small sample size. After this adjustment, AHI and CAI were no longer significantly different between the two groups.

Table 3 depicts the prevalence of various definitions of sleep apnea in both groups. Prevalence of both Elevated CAI and AASM CSA was significantly higher in the -HF group. Interestingly, when we used a pediatric CSA definition of CAI+HI $>1 / \mathrm{hr}$., the prevalence of pediatric CSA was similar in both groups.

CAI and OAI did not correlate with LVEF or any other echocardiogram z-score parameters.

Due to the wide range of CAI in the study population, we performed logarithmic transformation and depicted the relationship between LogCAI and age in Figure 2. CAI was inversely correlated to age at the time of sleep study (Pearson correlation coefficient $-0.245, \mathrm{p}=0.022$ ). OAI did not correlate with age (data not shown).

Using our definition of elevated central apnea index (CAI $>1 / \mathrm{hr}$.), we divided our entire cohort into two groups: normal CAI and elevated CAI. Table 4 compares these two groups' demographics, medical diagnoses, echocardiographic findings, and PSG findings. Children with elevated CAI were younger and had higher prevalence of prematurity. Fewer patients with structural heart disease or evidence of HF had elevated CAI. There was no statistically significant difference in LVEF or other echocardiographic parameters between the two groups.

\section{DISCUSSION}

Our study is the first to compare prevalence of CSA in children with and without HF. Contrary to adults, using the pediatric definition, prevalence of CSA is similar in children with and without HF. After adjusting for age, frequency of central apneic events did not differ between the two groups. Instead, CAI correlated inversely to age at time of sleep study. Children with elevated CAI were younger and had a higher prevalence of prematurity. Unlike the adult population, in children, CAI is not associated with LVEF.

We compared our results with the only other study on sleep disordered breathing in children with $\mathrm{HF}^{5}$. This was a prospective observational study, where the prevalence of CSA was $19 \%$ in children with HF secondary to dilated cardiomyopathy. This study used the pediatric CSA definition of central apnea/hypopnea per hour of sleep $>1$ as abnormal. Using similar criteria of pediatric CSA definition, prevalence of pediatric CSA in the +HF group of our study was high at $78.9 \%$ compared to the den Boer study (19\%) (Table 3). However, the children with $+\mathrm{HF}$ in our study were younger with a median age of 24 months compared to the den Boer study with a median age of 11.1 years. Using the same definition for pediatric CSA as the den Boer study (CAI+HI $>1 / \mathrm{hr}$., with $>50 \%$ of events being central), the prevalence of CSA did not differ between children with and without HF in our study. If we only compared the CAI in the two groups, it was found to be significantly lower in the $+\mathrm{HF}$ group. After adjusting for age, CAI was no longer different between the two groups. Thus, we conclude that, unlike the adult population, central apneic events were not increased in children with HF. Instead, central apneic events were more commonly seen in younger aged children, regardless of cardiac function.

Our results support previous findings, which did not show any correlation between AHI and severity of cardiac dysfunction measured by $\mathrm{LVEF}^{5,13}$. One study focused on assessing frequency of sleep disordered 
breathing and its relationship to cardiac function in children with cardiomyopathy found significant correlations between $\mathrm{CAI}+\mathrm{HI}$ and both $\mathrm{LV}$ end diastolic volume index and $\mathrm{LV}$ end systolic volume index ${ }^{13}$. We did not have data on LV end diastolic volume or end systolic volume index. 2D LV End-diastolic Septal Thickness vs BSA z-score and 2D LV End-systolic Dimension vs BSA z-score were higher in the + HF group. The septal thickness being significantly higher is not an intuitive result as the assumption is that dilated, poorly functioning hearts have a thinner septal thickness. With the presence of a subject with hypertrophic cardiomyopathy in conjunction with a small $\mathrm{n}$ of the $+\mathrm{HF}$ group showing a wide range up to a $\mathrm{z}$-score of 19.34, this was most likely enough to skew the average to significance. However, these parameters did not correlate with CAI or OAI. This finding contrasts the adult literature, where sleep disordered breathing is associated with left ventricular remodeling ${ }^{18}$. However, this is supported by previous studies in pediatric literature which suggests that pediatric sleep disordered breathing is not associated with significant cardiovascular strain and the majority of cardiovascular parameters in children with sleep disordered breathing are within the normal range at baseline ${ }^{19}$.

The relationship between age and CAI demonstrated in our study is intriguing and a key finding for future research in this field. In the previous study by den Boer, the median age of the 7 children with pediatric CSA was 2.9 years compared to 30 patients without CSA who had a median age of 12.3 years ${ }^{5}$. One of the biggest challenges of pediatric CSA is to have a consistent definition. While some authors have defined CSA as $\mathrm{CAI}+\mathrm{HI}>1 / \mathrm{hr}$., many have used a cut off of $5 / \mathrm{hr}$. While healthy term infants have an estimated median CAI of 5.5/hr. at 1 month of age ${ }^{20}$, older children aged 7.3 (4) years of age with Chiari 1 malformation had median CAI of $2.4(0.63-8.95)^{21}$. In our cross-sectional study, we found that CAI is inversely correlated to age. This has previously been described in children with trisomy $21^{22}$. Infants are particularly vulnerable to sleep apnea due to their upper airway structure ${ }^{23}$, ventilatory control ${ }^{24}$, arousal threshold ${ }^{25}$, laryngeal chemoreflex ${ }^{26}$, REM-predominant sleep state distribution ${ }^{27}$ and physiologically exaggerated laryngeal chemoreflexes that actively induce protective apneas ${ }^{28}$. Central respiratory pauses from immaturity of control of breathing are frequent during REM sleep in infants ${ }^{20}$.

While there are no studies exploring the relationship between age and central apnea, there are a few possible physiologic explanations involving central and carotid body chemoreception sensitivities. Thus far, chemoreceptor responsiveness has been evaluated in animal and cellular models ${ }^{29,30}$. However, the actual implications of this as a causative reason for the relationship between age and central apnea is still grounds for speculation and requires investigation with future study.

The primary limitation of our study is its retrospective nature. Hypopneas scored in the study could be obstructive or central in nature, thereby elevating CAHI and overestimating prevalence of CSA. We did not have data on medications used in heart failure which could also affect control of breathing. We did not have data on Cheyne Stoke breathing. Prospective cohort studies correlating objective measurements of ejection fraction to polysomnographic parameters are needed to gain a better understanding of the relationship between heart failure and CSA. Due to the cross-sectional nature of the study, we were unable to prove causality. Longitudinal studies can provide insight regarding the relationship between central apnea index and age. Finally, this is the experience of a single center, and practices may vary between different centers. This study is a pilot initiative to gain a better understanding of this patient population.

Ultimately, unlike adults, after adjusting for age, there is no difference in frequency of central apneic events in children with and without HF. CAI appears to be a function of age in children, rather than a function of ejection fraction, with younger patients demonstrating higher CAIs. Future studies exploring the relationship between CAI and age can gain a better understanding of determinants of CSA in children.

\section{REFERENCES}

1. Geib, T., Plappert, N., Roth, T., Popp, R., Birner, C., Maier, L.S., Pfeifer, M., and Arzt, M. (2015). Prevalence of Sleep-Disordered Breathing-Related Symptoms in Patients with Chronic Heart Failure and Reduced Ejection Fraction. Can J Cardiol 31, 839-845.

2. Javaheri, S., Shukla, R., Zeigler, H., and Wexler, L. (2007). Central sleep apnea, right ventricular 
dysfunction, and low diastolic blood pressure are predictors of mortality in systolic heart failure. J Am Coll Cardiol 49, 2028-2034.

3. Pierpont, M.E., Basson, C.T., Benson Jr, D.W., Gelb, B.D., Giglia, T.M., Goldmuntz, E., McGee, G., Sable, C.A., Srivastava, D., and Webb, C.L. (2007). Genetic basis for congenital heart defects: current knowledge: a scientific statement from the American Heart Association Congenital Cardiac Defects Committee, Council on Cardiovascular Disease in the Young: endorsed by the American Academy of Pediatrics. Circulation 115, 3015-3038.

4. Kay, J.D., Colan, S.D., and Graham, T.P. (2001). Congestive heart failure in pediatric patients. Am Heart J 142, 923-928.

5. den Boer, S.L., Joosten, K.F.M., van den Berg, S., Backx, A.P.C.M., Tanke, R.B., Sarvaas, G.J.d.M., Helbing, W.A., Rammeloo, L.A.J., ten Harkel, A.D.J., van Iperen, G.G., et al. (2016). Prospective Evaluation of Sleep Apnea as Manifestation of Heart Failure in Children. Pediatr Cardiol 37, 248-254.

6. Coniglio, A.C., and Mentz, R.J. (2020). Sleep Breathing Disorders in Heart Failure. Heart Fail Clin 16, $45-51$.

7. Javaheri, S., and Dempsey, J.A. (2013). Central sleep apnea. Compr Physiol 3, 141-163.

8. Oldenburg, O., Lamp, B., Faber, L., Teschler, H., Horstkotte, D., and Töpfer, V. (2007). Sleep-disordered breathing in patients with symptomatic heart failure A contemporary study of prevalence in and characteristics of 700 patients. Eur J Heart Fail 9, 251-257.

9. BRADLEY, T.D., TKACOVA, R., HALL, M.J., ANDO, S.-i., and FLORAS, J.S. (2003). Augmented sympathetic neural response to simulated obstructive apnoea in human heart failure. Clin Sci 104, 231-238.

10. Javaheri, S. (1999). A mechanism of central sleep apnea in patients with heart failure. N Engl J Med 341, 949-954.

11. Hall, M.J., Xie, A., Rutherford, R., Ando, S., Floras, J.S., and Bradley, T.D. (1996). Cycle length of periodic breathing in patients with and without heart failure. Am J Respir Crit Care Med 154, 376-381.

12. Combs, D., Skrepnek, G., Seckeler, M.D., Barber, B.J., Morgan, W.J., and Parthasarathy, S. (2018). Sleep-Disordered Breathing is Associated With Increased Mortality in Hospitalized Infants With Congenital Heart Disease. J Clin Sleep Med 14, 1551-1558.

13. Al-Saleh, S., Kantor, P.F., Chadha, N.K., Tirado, Y., James, A.L., and Narang, I. (2014). Sleepdisordered breathing in children with cardiomyopathy. Ann Am Thorac Soc 11, 770-776.

14. Berry, R.B., Budhiraja, R., Gottlieb, D.J., Gozal, D., Iber, C., Kapur, V.K., Marcus, C.L., Mehra, R., Parthasarathy, S., and Quan, S.F. (2012). Rules for scoring respiratory events in sleep: update of the 2007 AASM manual for the scoring of sleep and associated events. J Clin Sleep Med 8, 597-619.

15. McLaren, A.T., Bin-Hasan, S., and Narang, I. (2019). Diagnosis, management and pathophysiology of central sleep apnea in children. Paediatr Respir Rev 30, 49-57.

16. Sluysmans, T., and Colan, S.D. (2009). Structural measurements and adjustment for growth. Echocardiography in Pediatric and Congenital Heart Disease: From Fetus to Adult, 51-62.

17. Colan, S.D. (2016). Normal echocardiographic values for cardiovascular structures. Echocardiography in pediatric and congenital heart disease: from fetus to adult, 883-901.

18. Alonderis, A., Raskauskiene, N., Gelziniene, V., Mickuviene, N., and Brozaitiene, J. (2017). The association of sleep disordered breathing with left ventricular remodeling in CAD patients: a cross-sectional study. BMC Cardiovasc Disord 17, 250. 
19. Ehsan, Z., Ishman, S.L., Kimball, T.R., Zhang, N., Zou, Y., and Amin, R.S. (2017). Longitudinal cardiovascular outcomes of sleep disordered breathing in children: a meta-analysis and systematic review. Sleep 40.

20. Daftary, A.S., Jalou, H.E., Shively, L., Slaven, J.E., and Davis, S.D. (2019). Polysomnography Reference Values in Healthy Newborns. Journal of clinical sleep medicine : JCSM : official publication of the American Academy of Sleep Medicine 15, 437-443.

21. Amin, R., Sayal, P., Sayal, A., Massicote, C., Pham, R., Al-Saleh, S., Drake, J., and Narang, I. (2015). The Association between Sleep-Disordered Breathing and Magnetic Resonance Imaging Findings in a Pediatric Cohort with Chiari 1 Malformation. Can Respir J 22, 831569.

22. Fan, Z., Ahn, M., Roth, H.L., Li, L., and Vaughn, B.V. (2017). Sleep Apnea and Hypoventilation in Patients with Down Syndrome: Analysis of 144 Polysomnogram Studies. Children (Basel) 4, 55 .

23. Arens, R., and Marcus, C.L. (2004). Pathophysiology of upper airway obstruction: a developmental perspective. Sleep 27, 997-1019.

24. Gaultier, C., and Gallego, J. (2005). Development of respiratory control: evolving concepts and perspectives. Respir Physiol Neurobiol 149, 3-15.

25. Horne, R.S., Parslow, P.M., and Harding, R. (2005). Postnatal development of ventilatory and arousal responses to hypoxia in human infants. Respir Physiol Neurobiol 149, 257-271.

26. Reix, P., St-Hilaire, M., and Praud, J.P. (2007). Laryngeal sensitivity in the neonatal period: from bench to bedside. Pediatr Pulmonol 42, 674-682.

27. Curzi-Dascalova, L., Peirano, P., and Morel-Kahn, F. (1988). Development of sleep states in normal premature and full-term newborns. Dev Psychobiol 21, 431-444.

28. Davies, A., Koenig, J., and Thach, B. (1988). Upper airway chemoreflex responses to saline and water in preterm infants. J Appl Physiol 64, 1412-1420.

29. Darnall, R.A. (2010). The role of $\mathrm{CO}(2)$ and central chemoreception in the control of breathing in the fetus and the neonate. Respir Physiol Neurobiol 173, 201-212.

30. Gauda, E.B., Carroll, J.L., and Donnelly, D.F. (2009). Developmental maturation of chemosensitivity to hypoxia of peripheral arterial chemoreceptors-invited article. In Arterial Chemoreceptors. (Springer), pp 243-255.

\section{Hosted file}

Table 1.docx available at https://authorea.com/users/355844/articles/478945-heart-failureis-not-a-determinant-of-central-apnea-index-in-the-pediatric-population

\section{Hosted file}

Table 2.docx available at https://authorea.com/users/355844/articles/478945-heart-failureis-not-a-determinant-of-central-apnea-index-in-the-pediatric-population

\section{Hosted file}

Table 3.docx available at https://authorea.com/users/355844/articles/478945-heart-failureis-not-a-determinant-of-central-apnea-index-in-the-pediatric-population

\section{Hosted file}

Table 4.docx available at https://authorea.com/users/355844/articles/478945-heart-failureis-not-a-determinant-of-central-apnea-index-in-the-pediatric-population 


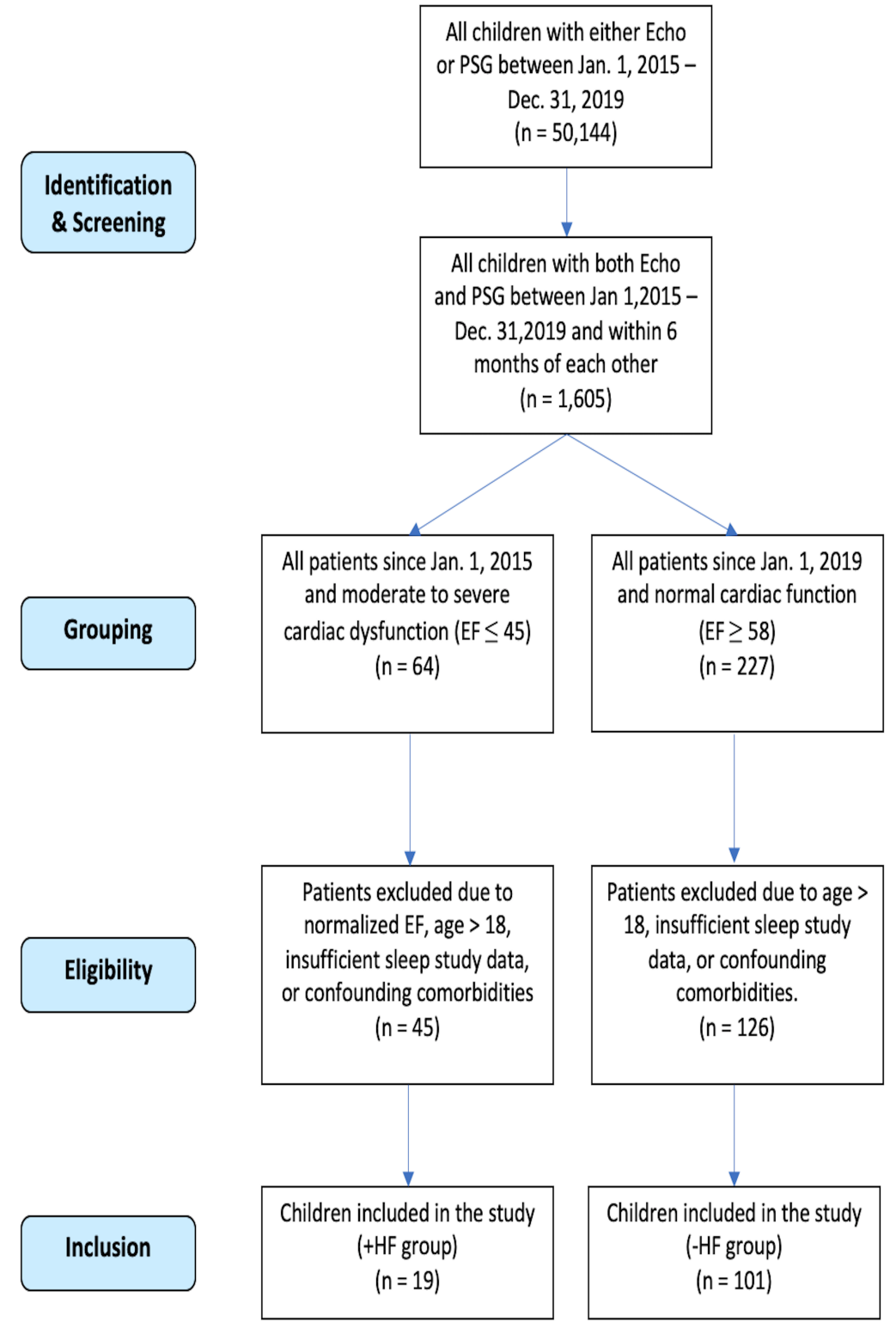




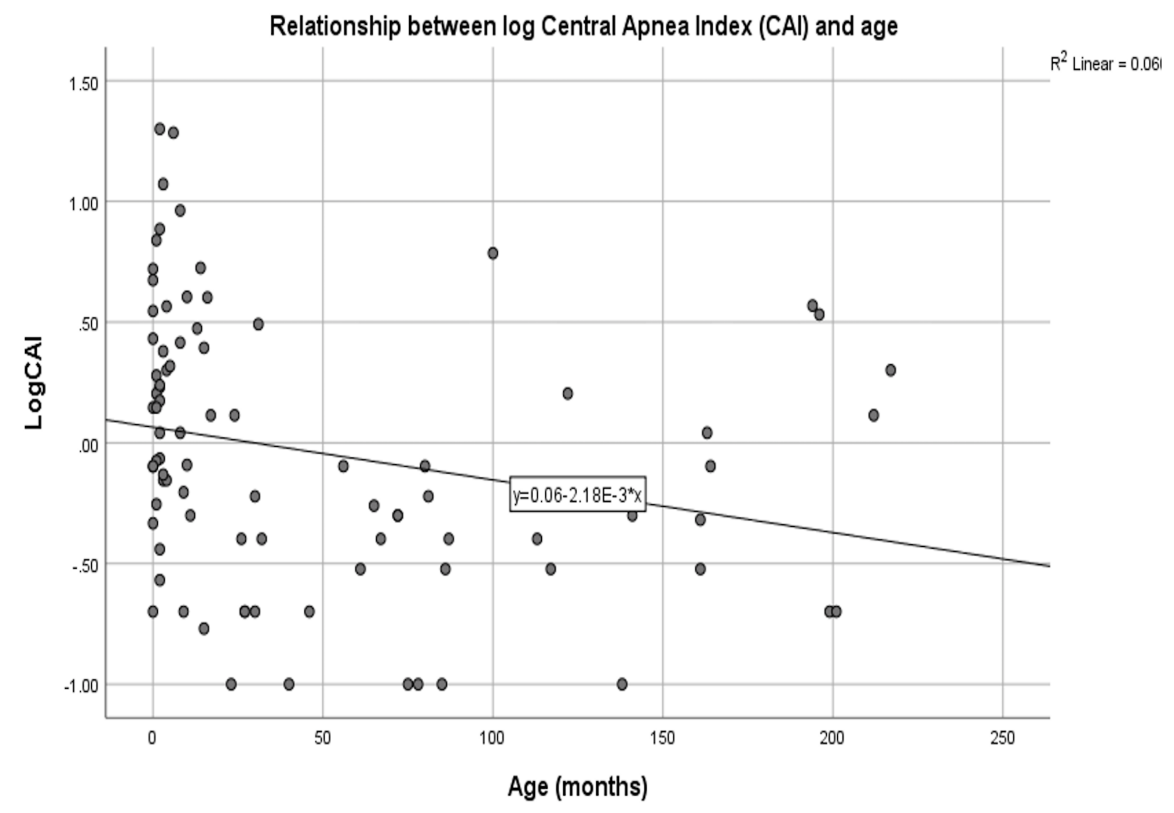

